\title{
Solderable and electroplatable flexible electronic circuit on a porous stretchable elastomer
}

\author{
Gi Seok Jeong ${ }^{1, \star}$, Dong-Hyun Baek ${ }^{1,2, \star}$, Ha Chul Jung ${ }^{1}$, Ji Hoon Song ${ }^{1}$, Jin Hee Moon ${ }^{1}$, Suck Won Hong ${ }^{3}$, \\ In Young $\mathrm{Kim}^{4}$ \& Sang-Hoon Lee ${ }^{1}$
}

A variety of flexible and stretchable electronics have been reported for use in flexible electronic devices or biomedical applications. The practical and wider application of such flexible electronics has been limited because commercial electronic components are difficult to be directly integrated into flexible stretchable electronics and electroplating is still challenging. Here, we propose a novel method for fabricating flexible and stretchable electronic devices using a porous elastomeric substrate. Pressurized steam was applied to an uncured polydimethylsiloxane layer for the simple and cost-effective production of porous structure. An electroplated nickel anchor had a key role in bonding commercial electronic components on elastomers by soldering techniques, and metals could be stably patterned and electroplated for practical uses. The proposed technology was applied to develop a plaster electrocardiogram dry electrode and multi-channel microelectrodes that could be used as a long-term wearable biosignal monitor and for brain signal monitoring, respectively.

\footnotetext{
${ }^{1}$ Department of Biomedical Engineering, College of Health Science, Korea University, Jeongneung 3-dong, Seongbuk-gu, Seoul 136-703, Korea. ${ }^{2}$ School of Electrical Engineering, Korea University, Anam-dong, Seongbuk-gu, Seoul 136-713, Korea. ${ }^{3}$ Department of Nanomaterials Engineering, College of Nanoscience \& Nanotechnology, Pusan National University, Samrangjin-ro 1268-50, Samrangjin-eup, Miryang, Gyeongnam 627-706, Korea. 4 Department of Biomedical Engineering, Hanyang University, 222 WangSimni-ro, Seongdong-gu, Seoul 133-791, Korea. ${ }^{\star}$ These authors equally contributed to this paper. Correspondence and requests for materials should be addressed to S.-H.L. (email: dbiomed@korea.ac.kr).
} 
$\mathrm{T}$ echnological advances are leading to the development of personalized medicine, ubiquitous health monitoring and neuron-to-machine interfaces for prostheses ${ }^{1,2}$. The development of long-term wearable or implantable biosignal-measuring devices that behave as an integral part of the body presents an important challenge that must be overcome before realizing these goals. A key technical issue is the development of tissue-like wearable or implantable electronics that are tissue- and skin-compatible, resistant to body fluids, flexible and sufficiently stretchable to adapt to tissue and body motions while maintaining close contact with the curvature of skin or organs. To date, several flexible or stretchable electronics for biomedical or industrial applications ${ }^{3-11}$ have been developed. Metal thin-film deposition on a flexible substrate, such as polyimide (PI), has been a standard approach ${ }^{12-18}$, although the elasticity of PI is limited and thin metal patterns can tolerate only limited deformation before breaking. Metal deposition and patterning on polydimethylsiloxane (PDMS) has received attention because PDMS is stretchable and is broadly used in biomedical applications, which makes it appropriate for attaching to the skin or for implanting into the body and has been extensively applied in the biomedical field. Metal films on PDMS, however, can sustain only limited stretching, and the stable and durable deposition of metal onto PDMS surfaces poses a great challenge. Several ingenious solutions to this problem have been described, including an accordionlike structure ${ }^{18,19}$, a meandering-shaped thin metal conductor on the PDMS surface $3,12,17,19-21$, bonding of two-dimensional stretchable Si nanomembranes onto elastomeric PDMS slabs, or printing of elastic conductors comprising single-walled nanotubes on elastic substrates ${ }^{22-24}$. Although these technologies have contributed greatly to the development of flexible electronics, each of them still has their limits (for example, complicated fabrication processes, and direct bonding of commercialized components on highly stretching substrates).
Several modified materials have been tested for their utility in flexible electronics, including nanoporous gold films suffused with nanometer-sized clusters of a platinum $(\mathrm{Pt})$ salt $^{25}$, aligned singlewalled carbon nanotubes (SWCNT) ${ }^{26}$, concentrated silver nanoparticle inks ${ }^{27}$, dissolvable films of silk fibroin ${ }^{28}$ and elastic conductors comprising uniformly dispersed SWCNTs ${ }^{29}$. Many of these methods were developed to realize stretchable soft electronics that could accommodate large applied strains without fracturing. Recently, epidermis-like electronics, where several components, including electrodes, simple electronic devices, sensors, power supplies and communication devices could be integrated, were reported ${ }^{30}$. Although these methods are useful and ingenious, they are of limited utility for ready-made electronic components, and the device fabrication processes are not compatible with conventional soldering-based methods. Furthermore, the surfaces of electrodes for recording multiple biosignals from a human body or skin require good contact to maximize the signal-to-noise ratio. To this end, an electrode should be electroplated with a metal, such as Pt. However, Pt electroplating to stretchable electrodes is still challenging because electroplated $\mathrm{Pt}$ is mechanically brittle and weak under stretching. Besides, the biocompatibility and long-term usability of most flexible electronics have not been fully evaluated.

In this paper, we describe a novel and simple method for fabricating flexible and stretchable electronics and for direct bonding of electronic components on the highly stretchable substrate. In the proposed method, electrodes were patterned on a porous elastomeric substrate to a minimum line width of $50 \mu \mathrm{m}$, and the electrode could be stretched maximally up to $80 \%$ before losing its electrical conductivity. The electrodes could be bent beyond $150^{\circ}$ without disconnecting and significant degradation of conductivity, and they survived over 20,000 stretching cycles under $30 \%$ strain with $5 \%$ per second strain rate. The key feature of this approach was the use of a porous highly stretchable PDMS substrate

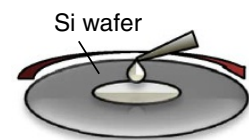

1.Spin coating prepolymer PDMS (thickness $=100 \sim 200 \mu \mathrm{m}$ )

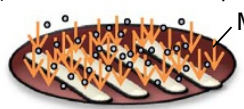

2.Steam etching

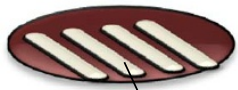

3.Porous PDMS membrane
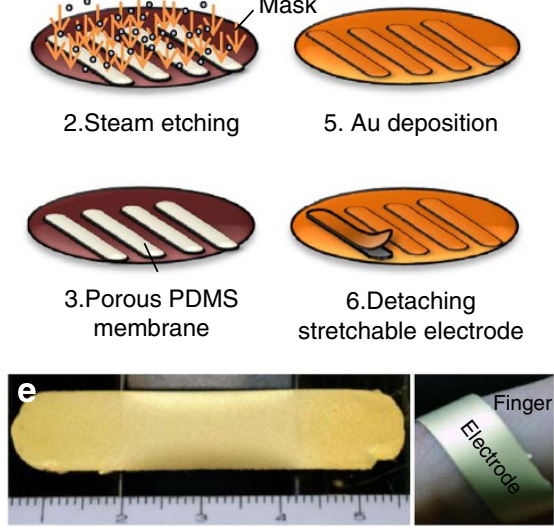

5. Au deposition

6.Detaching stretchable electrode b Steam
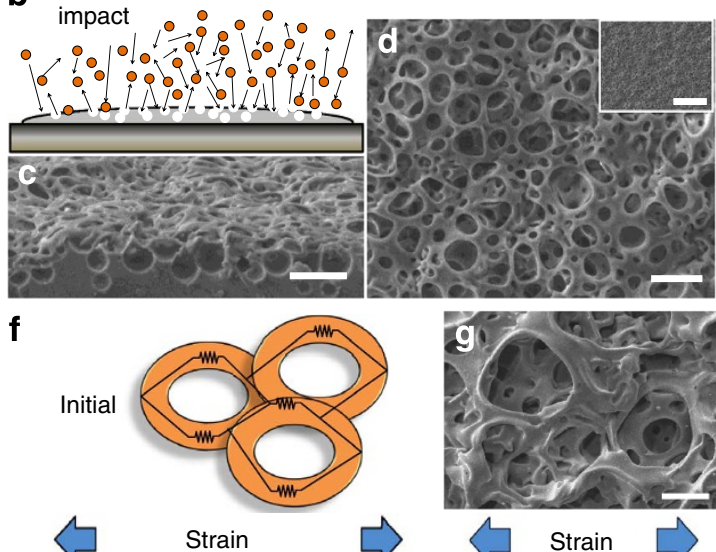

क
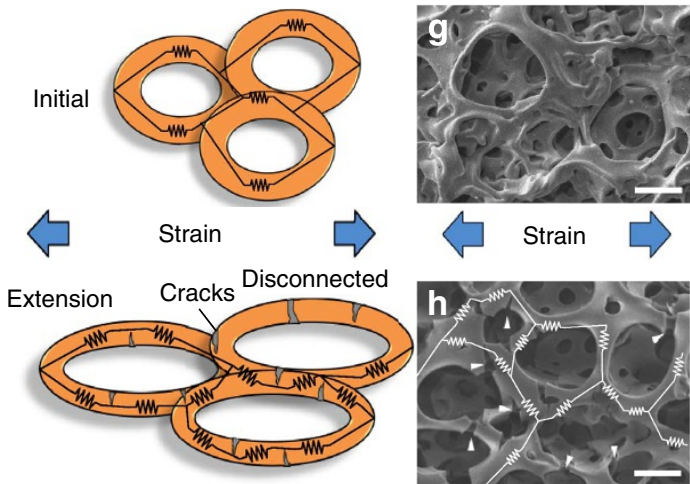

Figure 1 | Fabrication of the porous electrode with steam etching. (a) Process for fabricating a porous PDMS substrate using steam etching and metal (Au and Ti) deposition. (b) Schematic diagram showing the steam etching process. (c) A side-view of SEM image of a porous PDMS film. (d) Top view of SEM image of a porous PDMS film, and an inset showing a wide porous area. (e) Au- and Ti-deposited porous PDMS thin films (thickness: Ti (500 $\AA$ ), $\mathrm{Au}(2,000 \AA))$ and the winding of the film around a finger, maintaining close contact with the skin. (f) Schematic diagram showing the initial electrical connections and $(\mathbf{g})$ an SEM image of a metal-deposited porous layer before. (f) Schematic diagram showing the mechanism by which the structure stretched without incurring electrical disconnections (blue arrows: strain direction). (h) After 20,000 stretching cycles under $30 \%$ strain. (The white arrowheads indicate breaks in the structure after 20,000 stretching cycles under a 30\% strain, whereas white resistor symbols indicate intact conductive paths.) Scale bar, $20 \mu \mathrm{m}$ (c,d,g and $\mathbf{h})$ and $200 \mu \mathrm{m}$ (d, inset). 
a

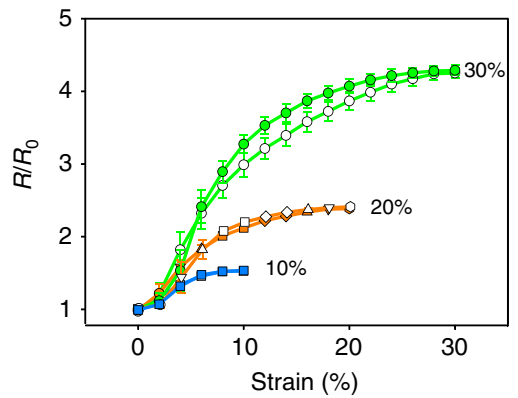

c

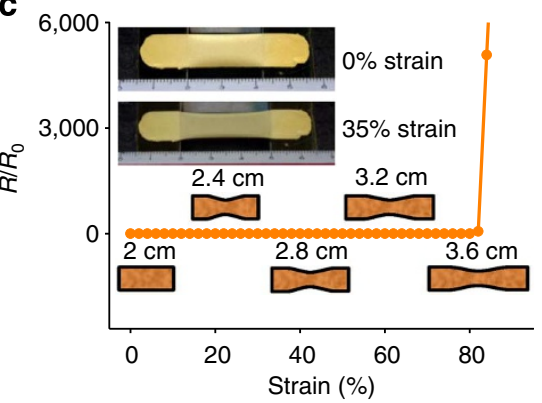

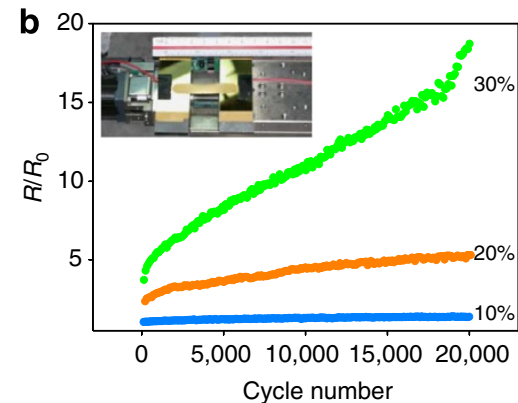

d

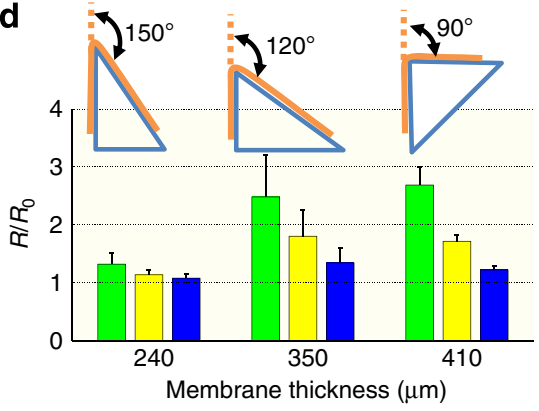

Figure 2 | Electrical and mechanical properties of the porous electrode. (a) Normalized electrical resistance on 10\% (blue line, s.d.: \pm 0.044 ), 20\% (orange line, s.d.: \pm 0.203 ) and $30 \%$ (green line, s.d.: \pm 0.289 ) stretching of the electrode for the analysis of hysteresis (empty symbols: contraction and full symbols: releasing). (b) Resistance changes during 20,000-cycle tests under $10 \%$ (blue), $20 \%$ (orange), or $30 \%$ (green) strains with $5 \%$ per second strain rate. The inset shows the reciprocating motion system set-up used to run the cycling tests. (c) Normalized resistance under the electrode stretching as a test for electrical disconnection (inset, stretched electrode with $35 \%$ strain). (d) Electrode bending tests for $150^{\circ}$ (green), $120^{\circ}$ (yellow), and $90^{\circ}$ (blue) bending along a sharp edge with different thickness of PDMS membranes (error bars indicate s.d.).

(pore size: $1-20 \mu \mathrm{m}$ ). A porous PDMS thin film was fabricated by applying pressurized steam to an uncured PDMS surface. The deposition and patterning of a metal thin film onto a porous PDMS substrate could be done with a patterned shadow mask. The metal electrodes were fabricated by electron beam deposition through a shadow mask to achieve microscale metal patterns. The porous structure stably supported the electroplated metal via metalelastomer interconnection. To confirm the biomedical applications and robustness of the patterned metal thin films on a PDMS surface, a test electrode was implanted inside of the body of a mouse for 2 months. A skin-like electrocardiogram (ECG) electrode was prepared, and its performance and skin compatibility were monitored over 1 week to evaluate its potential application for use in long-term ubiquitous biosignal monitoring. The influences of exercise and sweat on the electrode were also investigated. Multiple microelectrodes were fabricated and used to measure brain signals from the surface of a rat skull.

For the construction of an electronic circuit, the commercial electronic components need to be directly bonded on flexible substrates by soldering, however, direct bonding of a component on a highly stretchable substrate is still challenging. Although several methods to bond solid island circuits to the meandering-shaped thin metal conductor by soldering ${ }^{16-18,31}$ exist, they need to be encapsulated with PDMS. Thus a new paradigm is required for the direct bonding. We established a novel method for achieving direct leadfree soldering of commercial electrical components onto flexible substrates. These developments can be extended to flexible electronics applications and electrical skin-like devices.

\section{Results}

Fabrication of the porous PDMS thin layer and metal deposition. Large-area porous PDMS thin films were fabricated by applying steam to a PDMS prepolymer layer as illustrated in Fig. 1a (see Methods, and Supplementary Fig. S1 for detail). The depth of the porous structures could be controlled by the steam pressure and etching time. The porous PDMS thin film appeared to be semitransparent due to light scattering by the pores (Supplementary Fig. S2b). The porous PDMS layer is softer than the non-porous layer, and thermal expansion is almost similar (Supplementary Fig. S2c and Supplementary Tables S1 and S7). The effects of steam etching on the PDMS material properties were investigated by comparing the Fourier transform infrared spectroscopy spectrum to that of the untreated PDMS (cured on a hot plate for $2 \mathrm{~h}$ ) (Supplementary Fig. S3). The spectra were indistinguishable, indicating that the steam etching process did not affect the molecular structure of the PDMS. Figure $1 \mathrm{~b}$ shows a schematic diagram of the procedure used to generate the pores by steam impact, and Fig. 1c shows a sideview of scanning electron microscope (SEM) image of the porous PDMS layer. Figure 1d shows a top-view of SEM image of the porous PDMS surface, and the inset shows an SEM image of the wide porous area.

Layers of $\mathrm{Ti}(\sim 500 \AA$ thick $)$ and $\mathrm{Au}(\sim 2,000 \AA$ thick $)$ were successfully deposited onto the porous PDMS thin film (see Methods for detail). Figure 1e shows an image of the metal-deposited layer. The layer was sufficiently soft that it could be wrapped around a finger to produce close contact over the curvature of the surface without loss of the conductance properties. Figure $1 \mathrm{f}-\mathrm{h}$ illustrates a possible deformation mechanism that could explain the retention of conductivity under tension. As a tensile force was applied along the direction indicated by the arrow, the edges of the metalcoated pores became deformed, and some electrical pathways were broken; however, an electric pathway across the entire layer remained due to the interconnections, as clearly shown in the SEM images (Fig. 1h). Before the stretching test, the metal-deposited pores were round and symmetric. After 20,000 cycles under a 30\% strain, some of the metal-deposited pores broke (indicated by the white arrowheads in Fig. 1h); however, their electrical conductance was maintained. 
a

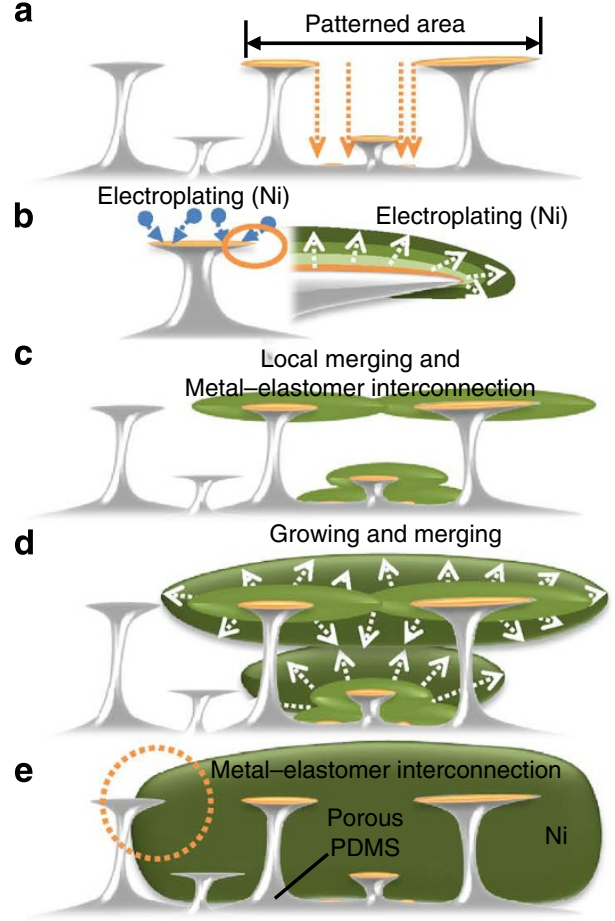

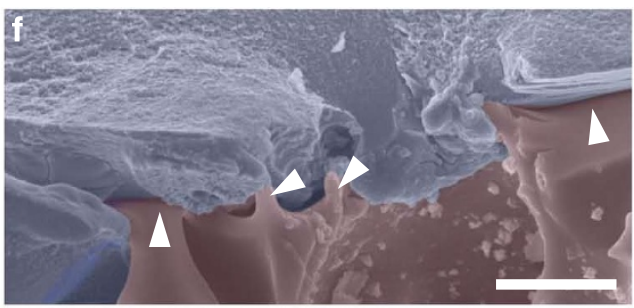
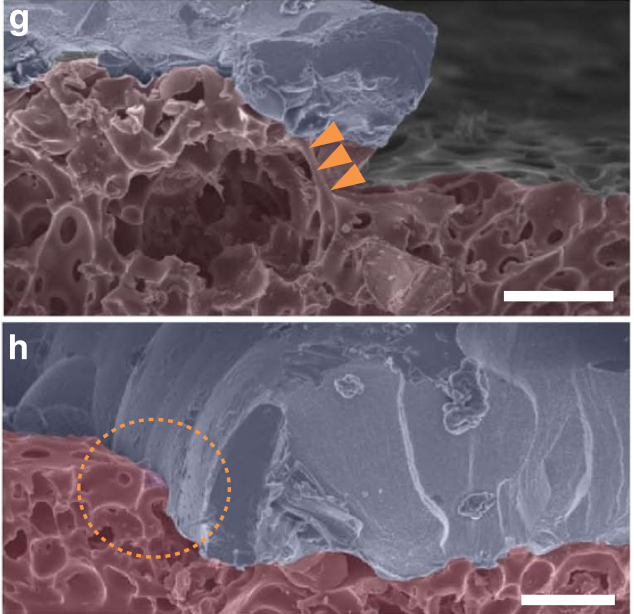

Figure 3 | Electroplating progress and mechanical coupling between Ni and porous PDMS surfaces. (a) Au (yellow areas) was deposited on the porous thin layer. (b) Ni was electroplated onto a gold pre-patterned surface (blue circles indicate Ni ions). (c,d) As the electroplating progressed, the Ni spread, merged horizontally and vertically, and (e) finally formed a Ni mass that could be used as an anchor for soldering (f) SEM images of Ni on the porous PDMS surface (the white arrowheads indicate the horizontal growth of $\mathrm{Ni}$ ). (g) SEM image of Ni growing vertically (orange arrowheads indicate the vertical coupling). (h) SEM image of the Ni anchor used for soldering; the orange circle indicates the region at which the porous PDMS and the Ni anchor were coupled. Blue and red areas indicate Ni and porous PDMS, respectively. Scale bar, $10 \mu \mathrm{m}(\mathbf{f})$ and $30 \mu \mathrm{m}(\mathbf{g}, \mathbf{h})$.

Mechanical reliability test. The metal layer deposited on the porous PDMS surface was tested for its usability as a stretchable soft electronic device. To this end, the normalized resistance $\left(R / R_{0}\right.$, where $R_{0}$ is the initial resistance and $R$ is the final resistance) was measured under application of an external force $\left(R_{0}\right.$ is shown in Supplementary Table S2). Electrical hysteresis in the stretchable electronics is important because electrical conductance should ideally be reproducible under strain cycling to maintain a high-accuracy operation ${ }^{25}$. The hysteresis of the metal on the PDMS layer was measured as a function of the stretching ratio (Fig. 2a; Supplementary Fig. S4). Although hysteresis was not observed during the 10 and $20 \%$ elongation tests, slight hysteresis was observed under $30 \%$ strain. To confirm the reliability of the metal-deposited porous PDMS thin film, a fatigue test was performed. Figure $2 \mathrm{~b}$ shows that at a $10 \%$ elongation, the normalized resistance change was less than $10 \%$ after 20,000 cycles (number of samples $=8$ ). Stretching by 20 and $30 \%$ increased the normalized resistance $\left(R / R_{0}\right)$. The maximum stretching capability of the metalized porous PDMS thin film was tested (Fig. 2c). The electrode could be stretched to an elongation of $80 \%$. Once the strain exceeded $80 \%$, the porous metal thin film was no longer conductive, although the electrode was not mechanically broken. The normalized resistance as a function of the bending angle was measured to demonstrate the electrical robustness under bending (Fig. 2d and Supplementary Fig. S5). The normalized resistance of the metal layer bent over the $150^{\circ}$ structure increased by $120 \%$ in $410 \mu \mathrm{m}$ thickness. The increase in the normalized resistance was less than $25 \%$ for a $90^{\circ}$ bending angle (Testing samples are represented in Supplementary Table S6).

Electroplating and soldering on the stretchable electric circuit. Stretchable and foldable electronics would be more broadly applicable if they were made to be electroplatable. Here, we tested $\mathrm{Ni}$ or Pt electroplating on the flexible electrodes. Ni electroplating was performed to create a metal anchor to affix commercialized electric components using soldering, whereas Pt electroplating was used to enhance the contact impedance. Figure $3 \mathrm{a}-\mathrm{e}$ show a schematic diagram in which the Ni electroplating on a gold-patterned area forms a mechanically robust anchor. The surface patterned with $\mathrm{Au}$ and Ti (Fig. 3a) was electroplated with a Ni layer that extended to cover the whole patterned area (Fig. 3b). As the Ni layer grew, electroplated $\mathrm{Ni}$ merged locally and horizontally to form a larger area (Fig. 3c). As growth continued, the vertically and horizontally growing Ni layers merged to form a larger cluster (Fig. 3d). Finally, a large $\mathrm{Ni}$ anchor formed on the porous PDMS layer (Fig. 3e). This Ni anchor adhered strongly to the surface due to metal (Ni)-elastomer interconnection, and the $\mathrm{Ni}$ anchor could be used to affix commercialized light-emitting diode (LED) cells using lead-free soldering techniques. Figure 3f,g demonstrate several coloured SEM images of the Ni electroplating process (Ni: blue, porous PDMS: red); Fig. $3 \mathrm{f}$ shows an image of the Ni layer (blue) on the porous PDMS surface (red, white arrowheads indicate metal-elastomer interconnections), and the orange arrowheads in Fig. $3 g$ indicate the vertically stretching porous structure of PDMS. The SEM image in Fig. $3 \mathrm{~h}$ shows a $\mathrm{Ni}$ anchor, and the orange circle indicates a robustly coupled region between the porous PDMS and the Ni anchor.

A microelectronic device was successfully fabricated on the porous PDMS surfaces using patterned metal deposition and electroplating processes. Figure 4 a shows the procedures used for metal patterning ( $\mathrm{Ti}$ and $\mathrm{Au}$ ), Ni electroplating and LED cell positioning, respectively. Individual commercial LED cells (HT-193NB $0603(16 \mathrm{~mm} \times 0.8 \mathrm{~mm})$, HARVATEK, Taiwan) were mounted and bonded to the electroplated $\mathrm{Ni}$ anchor using conventional soldering methods (Fig. 4b). Figure 4c shows line-and-space patterns on the 
a
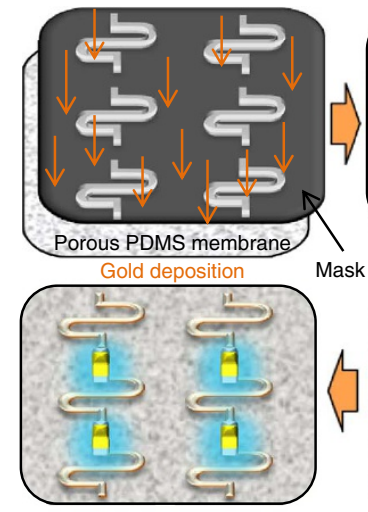

LED connection (Soldering)

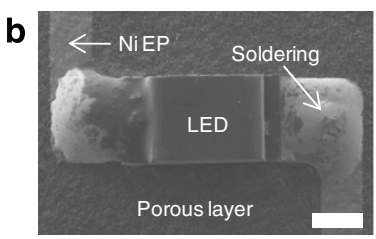

C

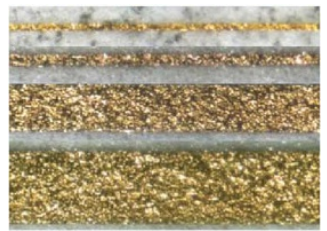

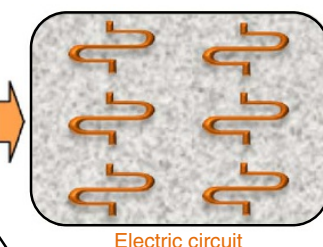

$\lcm{25}$

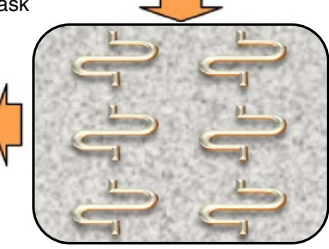

Electric circuit

(Ni electroplating)
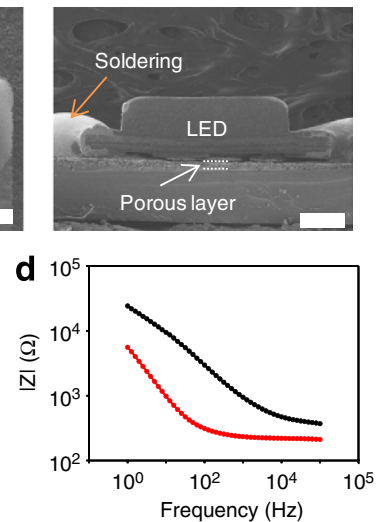
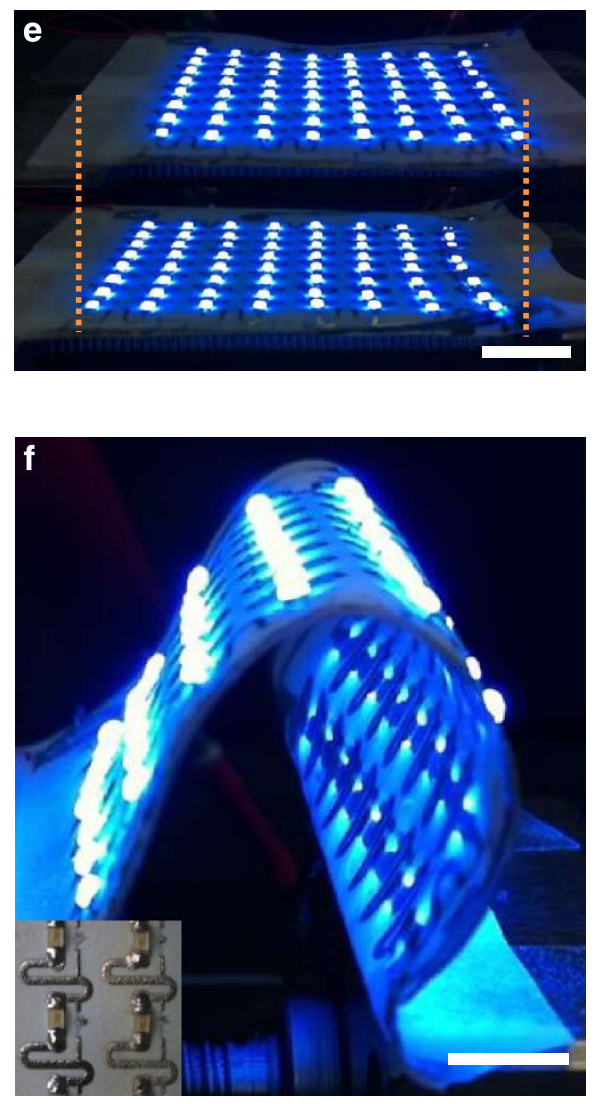

Figure 4 | Electroplating and soldering of the metal pattern onto a porous PDMS surface. (a) Procedures for metal patterning (Au and Ti), Ni electroplating and bonding of LEDs by soldering. (b) SEM image of commercial LEDs bonded to the metal pattern using conventional soldering methods. (c) Line patterns on the porous surface (50,100, 300 and $500 \mu \mathrm{m}$ ), with a minimum line width of $50 \mu \mathrm{m}$. The sheet resistance of a 3-cm Au line was $1.87 \pm 0.21 \Omega \square^{-1}$ (Supplementary Table S8). (d) Impedance of the Pt-electroplated (red) and non-electroplated electrodes (black). (e) Sixty-four shining LEDs before (top) and after (bottom) 24\% stretching (orange dotted lines represent stretching length). (f) Shining LEDs during repeated bending tests. The inset shows a photograph of LEDs and a circuit on a porous layer. Scale bar, $500 \mu \mathrm{m}$ (b-left), $300 \mu \mathrm{m}$ (b-right), $10 \mathrm{~mm}$ (e) and $5 \mathrm{~mm}(\mathbf{f})$.

porous PDMS surfaces with a minimum line width of $50 \mu \mathrm{m}$. The impedance of the Pt-electroplated or non-electroplated electrodes was measured using a potentiostat (EIS-300, Gamry) in a sinusoidal AC voltage mode $\left(20 \mathrm{mV}_{\mathrm{p}-\mathrm{p}}\right)$, and the results are plotted in Fig. $4 \mathrm{~d}$. In the low frequency range, the impedance of the Pt-electroplated electrodes (red) was dramatically enhanced. This frequency range encompasses the typical frequencies of biosignals, including electroencephalography and electromyography. A large number of LED cells $(8 \times 8)$ were manually bonded to the electroplated line using a conventional soldering iron. Figure $4 \mathrm{e}$ shows an image of 64 shining LEDs before (top) and after (bottom) application of 24\% stretching, indicating that all connections were stable and the light intensity did not decrease on stretching (Supplementary Movie 1). Figure $4 \mathrm{f}$ shows a photograph of the LEDs shining during the repeated bending tests (Supplementary Movie 2). The long-term repeating stretching test is shown in Supplementary Movies 3 and 4 (repeating cycles are shown in Supplementary Table S3). The 5\% cyclic stretching test demonstrated that the electroplated LED circuit maximally endured a maximum of 40,000 repeating cycles.

\section{Discussion}

In previous studies, the cost-effective and simple creation of highly stretchable and foldable electronics and bonding of commercial electronic components had been a significant challenge, which prevented more widespread applications to general electronics and biomedical areas. The proposed method above solved most of these problems using stretchable porous PDMS and Ni electroplating on metallic porous surfaces. The method to construct porous PDMS by pressurized steam is unique and advantageous for the mass production of porous structure on PDMS layer using a simple cost-effective process. The electrical conductivity of the metal patterns on the porous PDMS was robust under high extensions and after a durability test with over 20,000 cycles. Although the electrical conductivity was deteriorated owing to fatigue introduced by the repeated stretching motions, electrical disconnections were not observed. These results demonstrated that the porous electrodes maintained robust electrical conductance under stretching cycles (see Methods for detail). The electrode was also resistant to bending. Although the electrode was seriously bent along a sharp edge, electrical breakdown was not observed, indicating that the electrode was sufficiently flexible to withstand severe bending. The higher change in resistance based on the angle and thickness is clearly demonstrated in Supplementary Fig. S5. The metal layer is thin and deposited on the surface only. Therefore, for a larger bending angle, the thin metal layer expands much more. At the edge of the bend, the increase in bending angle causes a much larger extension of the thin metal layer (Supplementary Fig. S5a). As the thickness increases, the metal layer extends much more (Supplementary Fig. S5b). After all bending tests, the normalized resistance of the electrode regained its initial resistance, demonstrating the usability in foldable electronic devices.

A patterning of metal line on the porous elastomer layer and metal anchor to affix commercialized electric components was 
created by Ni electroplating, and multiple commercialized LEDs were bonded using conventional lead-free soldering techniques. All electrical circuits maintained their connections stably despite application of repeated bending and stretching. The inset shown in Fig. $4 \mathrm{f}$ presents a photograph of the interconnections and soldered regions. The interconnections remained stable and robust under deformation and stretching conditions (10-30\%), as observed by optical microscopy (Supplementary Fig. S6). To construct Ni anchor at specific location for soldering of LEDs, we electroplated Ni selectively by masking with manicure (Supplementary Fig. S7). To the best of our knowledge, direct soldering of commercial electrical components onto a highly flexible and stretchable elastomeric substrate has not previously been reported. Electrodes used to record biosignals are typically electroplated with Pt to enhance the contact impedance of the recording site; however, electroplated Pt is mechanically brittle, and enhancement of mechanical stability is important for achieving continuous biosignal measurements. We have electroplated Pt on the surface of porous elastomer as shown in Supplementary Fig. S8 for further extensive applications including the biomedical field. As predicted, the electroplated Pt was strongly adhered. A brief mechanical test (tape test) was performed to measure the mechanical stability of the Pt-electroplated layer; the photographs were taken after $0,1,5$ or 10 tape tests, and no critical changes or damages were observed, affirming a critical feature of the proposed method.

The potential and crucial application of this technology will be the biomedical fields including prosthetics, long-term continuous monitoring of biosignals and implantable electrodes. Figure $5 \mathrm{a}$ and Supplementary Movie 5 show a flexible metal-coated PDMS band attached to the skin of a finger, and an LED cell was connected by soldering. Under repeated extreme bending motions of the finger, the LEDs remained lit and changes in the brightness were not observed. These experiments indicated that the metal interconnections maintained excellent electrical conductivity, despite stretching and bending. We expect that a working electrical circuit could be built and used as a skin-like electronic device for the tether-free monitoring of biosignals or prosthetic devices, regardless of the presence of severe body motion. Two types of biosignal measurement electrodes were fabricated to demonstrate the potential of these fabrication techniques for biomedical applications. The first type of electrode was a plaster ECG electrode. We measured ECG signals using Ti- and Au-coated porous PDMS thin films, as shown in Fig. 5c. This electrode closely contacted the skin following the skin's curvature, like a plaster (Fig. 5e). The ECG signals were compared with those measured using a commercially available $\mathrm{Ag} / \mathrm{AgCl}$ electrode (HeartRode, HUREV, Korea) (Fig. 5d). As demonstrated in Fig. 5c, the P-wave, T-wave and QRS complex (PQRST waves) of the ECG were clearly observed, and the signals from the electrodes agreed well (Supplementary Fig. S9a,b). Over 1 week of continuous monitoring, the electrodes did not cause skin irritation or other problems (Fig. 5e; Supplementary Fig. S9c). To test the long-term wearability of the device, 10 recipients (Supplementary Table S4) wore the electrodes for 1 week, and the ECG signals were measured at 1, 3, 5 and 7 days. The signals for each day were not significantly degraded until the fifth day (Supplementary Fig. S10). On day 7 , the base line noise had noticeably increased and the amplitude of the $\mathrm{R}$ wave had decreased by about $30 \%$. As shown in Fig. 5c, motion artifacts and sweat effects, which previously presented the most significant challenges to dry electrode performance, were tested using our porous PDMS electrode. ECG signals were measured using our electrode and a commercial electrode mounted on a person at rest or walking $\left(5 \mathrm{~km} \mathrm{~h}^{-1}\right)$ (Fig. $\left.5 \mathrm{~d}\right)$. The performance of our electrode was robust in the presence of motion, and its signal was comparable to that of the commercial electrode, most likely owing to close contact between the electrode and the skin. The sweat effects were assessed after $30 \mathrm{~min}$ of exercise. Skin irritation was not observed after 1 week of continuous wearing.

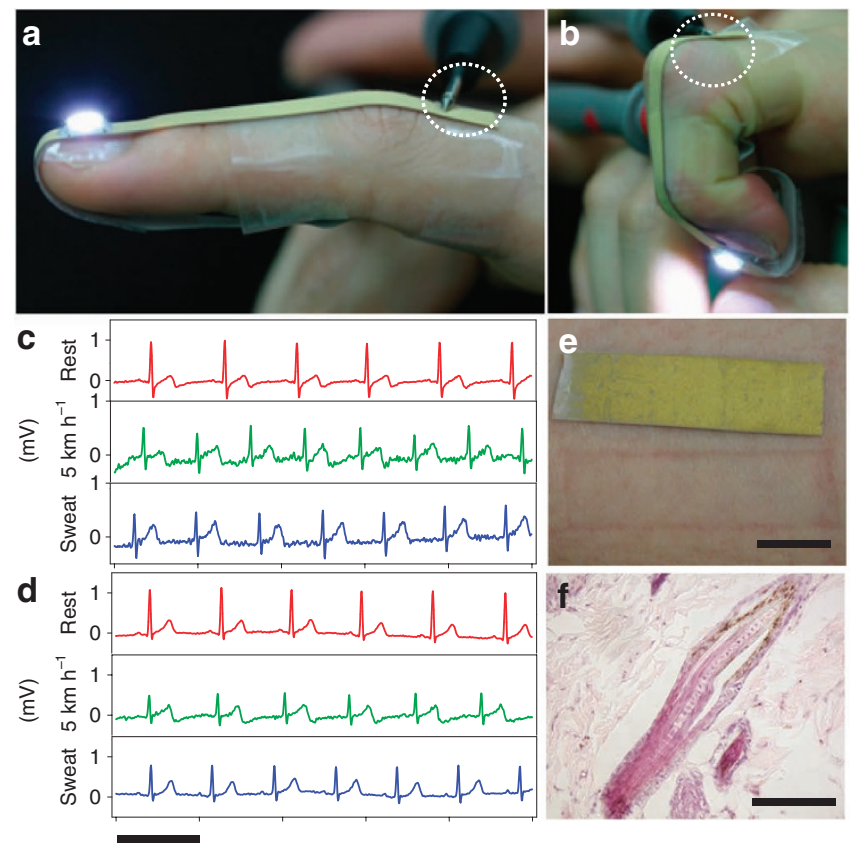

Figure 5 | Biosignal monitoring using skin-like electrode. (a) Skin electronics mounted on a finger. (b) Despite excessive finger bending, the LED shone brightly without experiencing a decrease in the light intensity (white dotted circle indicates an electric power connection). ECG signals recorded using (c) a Au-Ti-deposited plaster ECG electrode and (d) a commercial $\mathrm{Ag} / \mathrm{AgCl}$ electrode. $X$-axis scale bar indicates 1.0 s (red: resting, green: walking and blue: after exercise). (e) The skin after 1 week of continuously wearing the Au-Ti-deposited plaster ECG electrode; no skin irritation was observed. (f) Hematoxylin and eosin (H\&E) stained tissue after 8 weeks implantation of the electrode on the back of a mouse. Scale bars indicate $1 \mathrm{~cm}(\mathbf{e})$ and $100 \mu \mathrm{m}(\mathbf{f})$.

These electrodes may potentially be used for the long-term monitoring of other biosignals, such as electromyography and electroencephalography signals, which could enable the implementation of long-term wearable health monitoring systems. Flexible electrodes can also be used as implantable electrodes, as biocompatibility is an important consideration for long-term implantation in the body. To test biocompatibility, we implanted the electrode in the backs of mice for 8 weeks. At 1, 2, 4 and 8 weeks, the tissues were stained and visualized using hematoxylin and eosin (H\&E) (Fig. 5f; Supplementary Fig. S11). The tissue surrounding the electrode was normal, and inflammation was not observed. As a further application of our metal-deposited porous membrane, we fabricated an implantable electrode for measuring multi-channel neural signals. The impedance properties of such electrodes are important for measuring highquality signals in neural interface applications ${ }^{13,32,33}$. The recording site of the electrode was electroplated with $\mathrm{Pt}$, and patterned lines were insulated using PDMS. Figure 6a illustrates a multi-channel microelectrode fabricated to record epicranial brain signals from the skull of rat. To fabricate the electrode, the Au was electroplated on the recording sites $(20 \mu \mathrm{m}$ thickness $)$, and then Pt was electroplated successfully onto the recording site. Figure $6 \mathrm{~b}$ shows the position of the recording site. Channels 1 and 3 were located on the sensory area and channels 2 and 4 were located on the motor area. Figure $6 \mathrm{c}$ shows a photograph of an electrode fixed on the skull of a rat using dental cement. The electrode closely contacted on the surface along the rounded epicranial curvature. The measured signals were plotted, as shown in Fig. 6d. The hind paw was stimulated with a sharp needle to evoke a somatosensory potential response (channels 1 and 3). Even though the signals were measured from the 

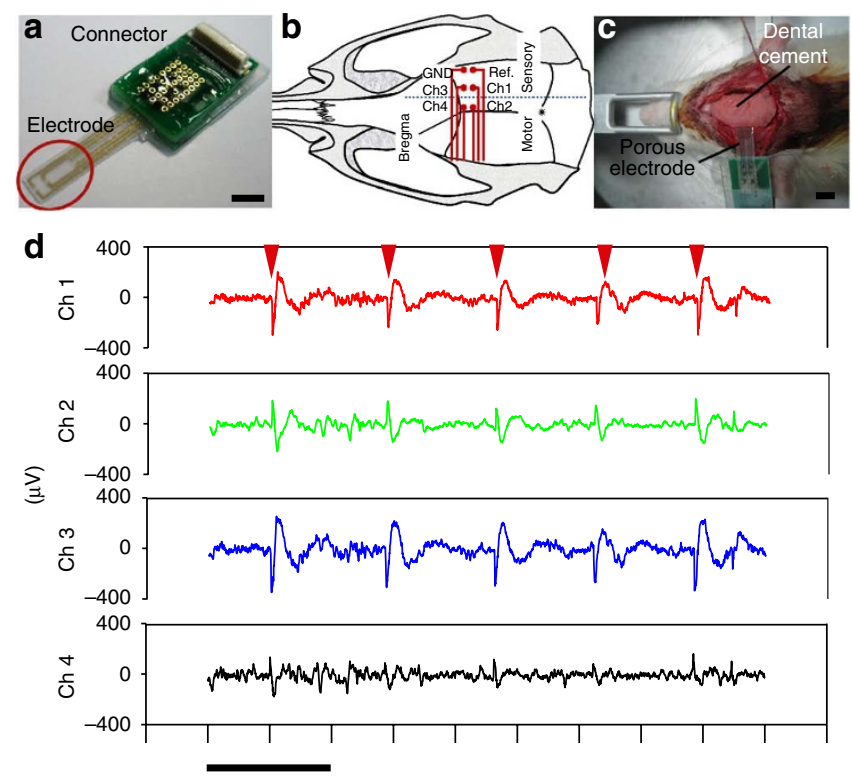

Figure 6 | Neurosignal monitoring. (a) The electrode used to measure an evoked potential signal from the skull of a rat. (b) The location of the recording site at which the signals were measured. (c) Electrode mounted on the skull of a rat and fixed using dental cement. (d) Evoked potential recorded using a porous PDMS electrode. The hind paw was stimulated using a sharp needle. (channel (Ch) 1 and 3 ) (red arrowheads). $X$-axis scale bar indicates $0.2 \mathrm{~s}$, and scale bars indicate $1.0 \mathrm{~cm}(\mathbf{a}, \mathbf{c})$

skull (red arrowheads in Fig. 6d) ${ }^{34,35}$. In contrast, the signal from channels 2 and 4 displayed a phase-reversal waveform typical of the motor area. The measured signal demonstrated that epicranial multi-channel recordings of evoked potentials could be obtained successfully, and the proposed electrode is useful for epicranial assessments of cortical activity in the brain. We used a total of three animals for sensing the epicranial brain signals.

In summary, biocompatible metal-patterned porous PDMS electronics with high flexibility and stretchability were successfully fabricated and adapted to conventional electrical circuit construction using simple and cost-effective techniques. The proposed technology may enable the preparation of foldable and stretchable electronic circuits, as would be useful in wearable electronics, prosthetic artificial limbs, sensory skins for robotics, body communication, haptic devices and wearable or implantable health monitoring devices. Biomedical applications require stable operation under a variety of conditions, and the materials must not induce skin or tissue incompatibility. Our method satisfied most of these requirements. The robustness of the Pt electroplating is another crucial feature of our method, and it will be widely useful for implantable neural electrodes. Such electrodes are useful in brain (or neuron)to-machine interfaces, which are under development in the context of prostheses that can ameliorate loss of sensory or motor function.

\section{Methods \\ Fabrication of the porous thin film. To fabricate the large-area plaster PDMS thin films, the PDMS precursor (a 10:1 mixture of the PDMS prepolymer and curing agent) was spin coated onto a Si wafer to a thickness of 100-200 $\mu \mathrm{m}$. Next, the prepared SU-8 shadow mask with a band shape was placed over the spin- coated PDMS prepolymer layer. The masked wafer was then placed inside a steam chamber and the uncured PDMS layer was exposed to pressurized steam provided by boiling water (Supplementary Fig. S1). Steam etching was performed for $5 \mathrm{~min}$, and the hot vapours simultaneously polymerized the PDMS to form solid porous structures (Fig. 1b; Supplementary Fig. S2a).}

Metal deposition. Ti and Au layers were deposited onto the porous structure using an e-beam evaporation process. Micro-scale metal patterning was performed by placing a Ni shadow mask on the porous layer, followed by e-beam deposition of a metal layer through the holes of the Ni mask. Finally, the porous metal-patterned layer was detached from the wafer by carefully pulling up.

Mechanical reliability test. The inset of Fig. $2 \mathrm{~b}$ shows the cycling test set-up, which consisted of a translational stage, a step motor and a control circuit for reciprocating the motion (5\% per second of strain rate, Supplementary Table S5). All the test processes were controlled by a computer system and programmed by Visual $\mathrm{C}++$. To demonstrate the electrical robustness under bending, support structures with angles of $150^{\circ}, 120^{\circ}$ or $90^{\circ}$ were prepared using an acryl block (Supplementary Fig. S5), and the metal layer on a porous PDMS surface was bent sharply over the edge of the angled structure. The resistance of the electrode was measured using a multimeter (Aglilent Technologies, HP 34401A Digital Multimeter, USA).

\section{References}

1. Teng, X. F., Zhang, Y. T., Poon, C. C. Y. \& Bonato, P. Wearable medical systems for p-health. IEEE Rev. Biomed. Eng. 1, 62-74 (2008).

2. Lebedev, M. A. \& Nicolelis, M. A. L. Brain-machine interfaces: past, present and future. Trends Neurosci. 29, 536-546 (2006).

3. Gray, D. S., Tien, J. \& Chen, C. S. High-conductivity elastomeric electronics. Adv. Mater. 16, 393-397 (2004).

4. Kim, D. H. et al. Materials for multifunctional balloon catheters with capabilities in cardiac electrophysiological mapping and ablation therapy. Nat. Materials 10, 316-323 (2011).

5. Rogers, J. A., Someya, T. \& Huang, Y. Materials and mechanics for stretchable electronics. Science 327, 1603 (2010).

6. Lacour, S. P. et al. Flexible and stretchable micro-electrodes for in vitro and in vivo neural interfaces. Med. Biol. Eng. Comput. 48, 945-954 (2010).

7. Ziaie, B. Stretchable bioelectrodes. Conf. Proc. IEEE Eng. Med. Biol. Soc. 1049-1052 (2009).

8. Ko, H. C. et al. A hemispherical electronic eye camera based on compressible silicon optoelectronics. Nature 454, 748-753 (2008).

9. Kim, K. et al. Large-scale pattern growth of graphene films for stretchable transparent electrodes. Nature 457, 706-710 (2009).

10. Takei, K. et al. Nanowire active-matrix circuitry for low-voltage macroscale artificial skin. Nat. Materials 9, 821-826 (2010).

11. Loher, T. et al. Stretchable electronic systems: realization and applications. Electronics Packaging Technology Conference EPTC, Singapore, 893-898 (2009).

12. Verplancke, R., Bossuyt, F., Cuypers, D. \& Vanfleteren, J. Thin-film stretchable electronics technology based on meandering interconnections: fabrication and mechanical performance. J. Micromech. Microeng. 22, 015002 (2012).

13. Baek, D. et al. Interconnection of multichannel polyimide electrodes using anisotropic conductive films (ACFs) for biomedical applications. IEEE Trans. Biomed. Eng. 58, 1466-1473 (2010).

14. Navarro, X. et al. A critical review of interfaces with the peripheral nervous system for the control of neuroprostheses and hybrid bionic systems. J. Peripher. Nerv. Syst. 10, 229-258 (2005).

15. Rousche, P. J., Pellinen, D. S., Pivin Jr, D. P., Williams, J. C. \& Vetter, R. J. Flexible polyimide-based intracortical electrode arrays with bioactive capability. IEEE Trans. Biomed. Eng. 48, 361-371 (2001).

16. Axisa, F., Bossuyt, F., Vervust, T. \& Vanfleteren, J. Laser based fast prototyping methodology of producing stretchable and conformable electronic systems. 2nd Electronics System Integration Technology Conference, Greenwich, London, UK, 1387-1390 (2008).

17. Brosteaux, D., Axisa, F., Gonzalez, M. \& Vanfleteren, J. Design and fabrication of elastic interconnections for stretchable electronic circuits. Electr. Device Lett. IEEE 28, 552-554 (2007).

18. Fjelstad, J. Flexible Circuit Technology 4th edn, Ch. 13 (Silicon Valley Publishers Group, 2011).

19. Loher, T. et al. Stretchable electronic systems. Electronics Packaging Technology Conference EPTC, Singapore 271-276 (2006).

20. Kim, D. H., Xiao, J., Song, J., Huang, Y. \& Rogers, J. A. Stretchable, curvilinear electronics based on inorganic materials. Adv. Mater. 22, 2108-2124 (2010).

21. Vieroth, R. et al. Stretchable circuit board technology and application. International Symposium on Wearable Computers, ISWC, Linz, Austria, 33-36 (2009).

22. Sekitani, T. et al. A rubberlike stretchable active matrix using elastic conductors. Science 321, 1468-1472 (2008).

23. Kim, D. H. et al. Materials and noncoplanar mesh designs for integrated circuits with linear elastic responses to extreme mechanical deformations. Proc. Natl Acad. Sci. 105, 18675-18680 (2008).

24. Choi, W. M. et al. Biaxially stretchable 'wavy' silicon nanomembranes. Nano Lett. 7, 1655-1663 (2007).

25. Urdaneta, M. G., Delille, R. \& Smela, E. Stretchable electrodes with high conductivity and photo-patternability. Adv. Mater. 19, 2629-2633 (2007).

26. Yamada, T. et al. A stretchable carbon nanotube strain sensor for humanmotion detection. Nat. Nanotechnol. 6, 296-301 (2011).

27. Ahn, B. Y. et al. Omnidirectional printing of flexible, stretchable, and spanning silver microelectrodes. Science 323, 1590-1593 (2009). 
28. Kim, D. H. et al. Dissolvable films of silk fibroin for ultrathin conformal bio-integrated electronics. Nat. Materials 9, 511-517 (2010).

29. Sekitani, T. et al. Stretchable active-matrix organic light-emitting diode display using printable elastic conductors. Nat. Materials 8, 494-499 (2009).

30. Kim, D. H. et al. Epidermal electronics. Science 333, 838-843 (2011).

31. Carta, R. et al. Design and implementation of advanced systems in a flexiblestretchable technology for biomedical applications. Sensor. Actuat. A Phys. 156, 79-87 (2009).

32. Abidian, M. R., Ludwig, K. A., Marzullo, T. C., Martin, D. C. \& Kipke, D. R. Interfacing conducting polymer nanotubes with the central nervous system: chronic neural recording using poly ( 3,4 ethylenedioxythiophene) nanotubes. Adv. Mater. 21, 3764-3770 (2009).

33. Franks, W., Schenker, I., Schmutz, P. \& Hierlemann, A. Impedance characterization and modeling of electrodes for biomedical applications. IEEE Trans. Biomed. Eng. 52, 1295-1302 (2005).

34. MacDonald, K. D. \& Barth, D. S. High frequency (gamma-band) oscillating potentials in rat somatosensory and auditory cortex. Brain Res. 694, 1-12 (1995).

35. Troncoso, E., Muller, D., Czellar, S. \& Zoltan Kiss, J. Epicranial sensory evoked potential recordings for repeated assessment of cortical functions in mice. J. Neurosci. Methods 97, 51-58 (2000).

\section{Acknowledgements}

This study was supported by a grant of the Korean Health Technology R\&D Project, Ministry of Health \& Welfare, Republic of Korea. (A092052), and the Public welfare \& Safety research program through the National Research Foundation of Korea (NRF) funded by the Ministry of Education, Science and Technology (2010-0020787).

\section{Author contribution}

G.S.J developed the porous membrane, proposed the key idea of this paper, designed and carried out the experiments, prepared most of the data and wrote the paper. D.H.B proposed the idea for the electronics, developed the metal deposition and patterning procedure, carried out the soldering experiments and animal experiment and prepared most of the data. H.C.J carried out the experiments for the fabrication of electrodes and animal experiment. J.H.S assisted with fabricating the porous membrane. J.H.M assisted with designing the electric circuits. S.W.H. gave ideas for the characterization of stretchable soft electronics and assisted in writing the paper; I.Y.K contributed in biosignal analysis, animal experiment and writing the paper; S.-H.L. proposed the key idea, managed the research process and wrote the paper.

\section{Additional information}

Supplementary Information accompanies this paper at http://www.nature.com/ naturecommunications

Competing financial interests: The authors declare no competing financial interests.

Reprints and permission information is available online at http://npg.nature.com/ reprintsandpermissions/

How to cite this article: Jeong, G. S. et al. Solderable and electroplatable flexible electronic circuit on a porous stretchable elastomer. Nat. Commun. 3:977 doi: $10.1038 /$ ncomms1980 (2012). 